\section{Probing the initiation and effector phases of the somatic piRNA pathway in Drosophila}

Astrid D. Haase, ${ }^{1}$ Silvia Fenoglio, ${ }^{1}$ Felix Muerdter, ${ }^{1}$ Paloma M. Guzzardo, ${ }^{1}$ Benjamin Czech, ${ }^{1}$ Darryl J. Pappin, ${ }^{1}$ Caifu Chen, ${ }^{2}$ Assaf Gordon, ${ }^{1}$ and Gregory J. Hannon ${ }^{1,3}$

\begin{abstract}
${ }^{1}$ Watson School of Biological Sciences, Howard Hughes Medical Institute, Cold Spring Harbor Laboratory, Cold Spring Harbor, New York 11724, USA; ${ }^{2}$ Genomic Assays R\&D Life

Technologies, Foster City, California 94404, USA
\end{abstract}

Combining RNAi in cultured cells and analysis of mutant animals, we probed the roles of known Piwi-interacting RNA (piRNA) pathway components in the initiation and effector phases of transposon silencing. Squash associated physically with Piwi, and reductions in its expression led to modest transposon derepression without effects on piRNAs, consistent with an effector role. Alterations in Zucchini or Armitage reduced both Piwi protein and piRNAs, indicating functions in the formation of a stable Piwi RISC (RNA-induced silencing complex). Notably, loss of Zucchini or mutations within its catalytic domain led to accumulation of unprocessed precursor transcripts from flamenco, consistent with a role for this putative nuclease in piRNA biogenesis.

Supplemental material is available at http://www.genesdev.org.

Received July 9, 2010; revised version accepted September 17, 2010.

Eukaryotic small RNAs regulate gene expression through various mechanisms, intervening at both transcriptional and post-transcriptional levels (for review, see Ghildiyal and Zamore 2009). Small RNAs are divided into classes according to their mechanism of biogenesis and their particular Argonaute protein partner. Piwi-interacting RNAs (piRNAs) bind Piwi-clade Argonaute proteins and act mainly in gonadal tissues to guard genome integrity by silencing mobile genetic elements (for review, see Malone and Hannon 2009).

Conceptually, the piRNA pathway can be divided into several different phases. During the initiation phase, small RNAs, called primary piRNAs, are produced from their generative loci, so-called piRNA clusters (Brennecke et al. 2007). These give rise to long, presumably single-stranded precursor transcripts, which are processed via an unknown biogenesis mechanism into small RNAs that are larger than canonical microRNAs ( 24-30 nucleotides [nt]) (Aravin et al. 2006; Girard et al. 2006; Grivna et al. 2006;

[Keywords: Piwi; Zucchini; Armitage; Squash; primary piRNA; Drosophila]

${ }^{3}$ Corresponding author.

E-MAIL hannon@cshl.edu; FAX (516) 367-8874.

Article published online ahead of print. Article and publication date are online at http://www.genesdev.org/cgi/doi/10.1101/gad.1968110.
Lau et al. 2006; Vagin et al. 2006). Primary piRNAs become stably associated with Piwi proteins to form Piwi RISCs (RNA-induced silencing complexes), which also contain additional proteins that facilitate target recognition and silencing. During the effector phase, Piwi RISCs identify targets via complementary base-pairing. In some cases, for example, with Aubergine as a piRNA partner, there is strong evidence for target cleavage in vivo (Brennecke et al. 2007; Gunawardane et al. 2007). This nucleolytic destruction of transposon mRNAs is probably the main Aubergine effector mechanism, although this has not been rigorously demonstrated. Piwi also conserves the Argonaute catalytic triad; however, in this case, both its nuclear localization and its association with certain chromatin proteins suggest the possibility of transcriptional and post-transcriptional effector pathways (Brower-Toland et al. 2007; Klattenhoff et al. 2009; Saito et al. 2009). An additional phase, adaptation, is restricted to germ cells and constitutes the ping-pong cycle. During this phase, transposon mRNA cleavage directed by primary piRNAs triggers the production of secondary piRNAs, whose $5^{\prime}$ ends correspond to cleavage sites (Brennecke et al. 2007; Gunawardane et al. 2007). These generally join Ago3 and enable it to recognize and cleave RNAs with antisense transposon content, perhaps piRNA cluster transcripts. Cleavage by Ago3 RISC again triggers piRNA production from the target, closing a loop that enables the overall small RNA population to adjust to challenge by a particular transposon (for review, see Aravin et al. 2007). Finally, piRNA populations present in germ cells can be transmitted to the next generation to prime piRNA responses in progeny (Brennecke et al. 2008).

In Drosophila follicle cells, only the initiation and effector phases appear relevant (Brennecke et al. 2008). Here, the piRNA pathway relies on the coupling between a single Piwi protein (Piwi itself) and a principal piRNA cluster (flamenco) to silence mainly gypsy family retrotransposons (Sarot et al. 2004; Saito et al. 2006; Brennecke et al. 2007; Li et al. 2009; Malone et al. 2009). Drosophila ovarian somatic sheet cells (OSS) display many of the properties of follicle cells, and represent a convenient system to study the initiation and effector phases of the piRNA pathway without the complications inherent in the study of complex tissues in vivo (Niki et al. 2006; Lau et al. 2009; Saito et al. 2009). We therefore sought to leverage information derived from the use of RNAi in OSS cells with the analysis of ovaries derived from mutant animals to probe the roles of known piRNA pathway components in the initiation and effector phases of transposon silencing.

\section{Results and Discussion}

The piRNA pathway is continuously required for transposon silencing

Several prior studies have proposed models in which Piwi proteins silence targets by interfering with their transcription (Pal-Bhadra et al. 2004). Since piRNAs are largely absent from somatic tissues (Cox et al. 2000), impacts underlying these changes are presumed to have occurred during development and to have been epigenetically maintained in the adult. Drosophila Piwi protein is mainly localized to the nucleus and has been shown to 
interact with $\mathrm{HP} 1$, a core component of heterochromatin (Pal-Bhadra et al. 2004; Brower-Toland et al. 2007). Considered together, this body of evidence pointed strongly to an effector mechanism in which Piwi-associated small RNAs direct heterochromatin formation and silencing of targets.

Loss of piwi has dramatic effects on transposon expression in somatic follicle cells (Sarot et al. 2004; Brennecke et al. 2007; Klenov et al. 2007; Malone et al. 2009). Genetic mutants result in an absence of Piwi protein throughout development. This could lead to a failure to create heterochromatic marks that could have otherwise maintained epigenetic silencing of transposons in the absence of continuous Piwi expression. Alternatively, there could be an ongoing requirement for Piwi to maintain silencing, irrespective of whether it acted via transcriptional or post-transcriptional mechanisms.

To discriminate between these possibilities, we transfected OSS cells with dsRNAs corresponding to piwi, and followed impacts on Piwi mRNA and protein levels (Supplemental Fig. S1A; data not shown). Maximal suppression was reached by $3 \mathrm{~d}$, and silencing persisted through day 6 . At 6 d post-transfection, we probed impacts on two elements known to be derepressed in the follicle cells of piwi mutant ovaries: gypsy and idefix. Both showed derepression (up to 10-fold) (Fig. 1A) upon piwi silencing. Additional elements were also tested (Supplemental Fig. S1B), with blood being impacted strongly. Previous studies have also implicated zucchini (zuc) in the function of the somatic piRNA pathway (Pane et al. 2007; Malone et al. 2009). RNAi against this gene also increased gypsy, blood, and idefix expression (Fig. 1A; Supplemental Fig. S1B). Considered together, these results demonstrate that the integrity of the piRNA pathway is essential for the ongoing repression of mobile elements and argue against a model in

A
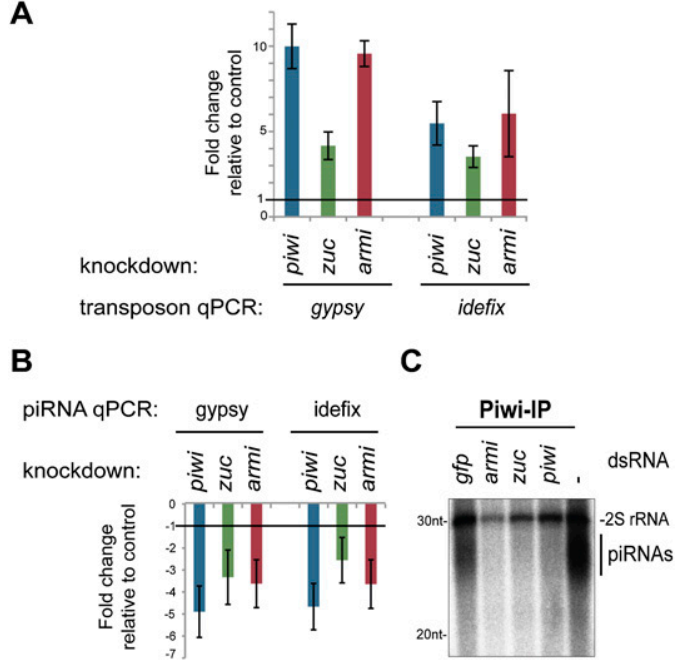

Figure 1. Effect of piwi, zucchini, and armitage knockdown on transposon silencing in OSS cells. OSS cells were treated with dsRNA against piwi, zuc, or armi for 6 d. qPCRs were normalized to internal controls $r p 49(A)$ or bantam $(B)$. Fold changes relative to cells treated with gfp-dsRNA are shown on a linear scale. Error bars represent one standard deviation over three biological replicates. Transcripts $(A)$ and two abundant piRNAs $(B)$ corresponding to gypsy and idefix retroelements were detected by qPCR. (C) Small RNAs coimmunoprecipitating with Piwi in untreated cells and cells treated with dsRNA against gfp, armi, zuci, or piwi were labeled with ${ }^{32} \mathrm{P}$ at their $5^{\prime}$ termini. which silent epigenetic states, once set by the action of piwi proteins on chromatin, can autonomously maintain transposon silencing.

Armitage is a component of the somatic piRNA pathway

Nearly a dozen proteins have been linked to the fully elaborated piRNA pathway that operates in germ cells (Malone et al. 2009). Many of these show germ cell-specific expression patterns consistent with their selective biological effects. Mutations in armitage (armi) result in coincident loss of the characteristic nuclear accumulation of Piwi protein and a reduction in Piwi-associated piRNAs (Malone et al. 2009). Unlike most germline-specific pathway components, an examination of RNA-seq data from OSS cells indicated substantial armi expression (Supplemental Fig. S1C). We therefore suppressed armi by RNAi and examined effects on transposon expression. Notably, gypsy, blood, and idefix were strongly derepressed, implying a role for armi in both the somatic and germline compartments (Fig. 1A; Supplemental Fig. S1B).

The Drosophila mutant armi ${ }^{1}$ represents a P-element insertion in the $5^{\prime}$ untranslated region (UTR) of armitage. A second allele, $\operatorname{armi}^{72.1}$, was derived from $a r m i{ }^{1}$ by imprecise excision (Cook et al. 2004). RNA-seq data covered the armi ORF in OSS, but no reads were detected corresponding to the germ cell 5' UTR (Supplemental Fig. S1D). This raises the possibility that armi expression might be driven by an alternative promoter in somatic cells, and that the armi alleles examined thus far may have spared the activity of that promoter.

\section{Armitage and Zucchini function at the initiation phase}

To investigate whether Armi and Zuc act at the initiation or effector phase of the piRNA pathway, we examined piRNAs. Silencing of piwi reduced levels of two abundant piRNAs, corresponding to gypsy, or idefix (Fig. 1B). Similar effects were noted upon silencing of armi or zuc. Aggregate OSS piRNA levels can be measured qualitatively by radioactive phosphate exchange of small RNAs in Piwi immunoprecipitates. As expected, RNAi against piwi virtually eliminated piRNAs in immunoprecipitates (Fig. 1C). Silencing of armi or zuc produced indistinguishable effects.

In germ cells, armi mutation causes loss of the prominent nuclear localization of Piwi (Malone et al. 2009). We observed a similar phenotype upon knockdown of armi in somatic OSS cells (Fig. 2A). Because of the mixed cell types present in ovaries, previous studies had not been able to distinguish whether Armi loss simply caused Piwi mislocalization or whether Armi influenced Piwi expression or stability. In OSS cells, knockdown of armi reduced Piwi protein levels by approximately fivefold, equivalent to a targeted knockdown of Piwi itself without affecting piwi mRNA (Fig. 2B,C). We noted a similar loss of Piwi protein from the nuclei in cells exposed to zuc-dsRNAs (Fig. 2A). In this case, Piwi protein but not mRNA levels also fell (Fig. 2B,C).

Considered together, these data strongly suggest roles of Armi and Zuc in the initiation phase of the piRNA pathway. A role for Armi, along with a previously unrecognized component, $\mathrm{Yb}$, in the somatic pathway, is also supported by a recent report from Brennecke and colleagues (Olivieri et al. 2010). Either protein could play 


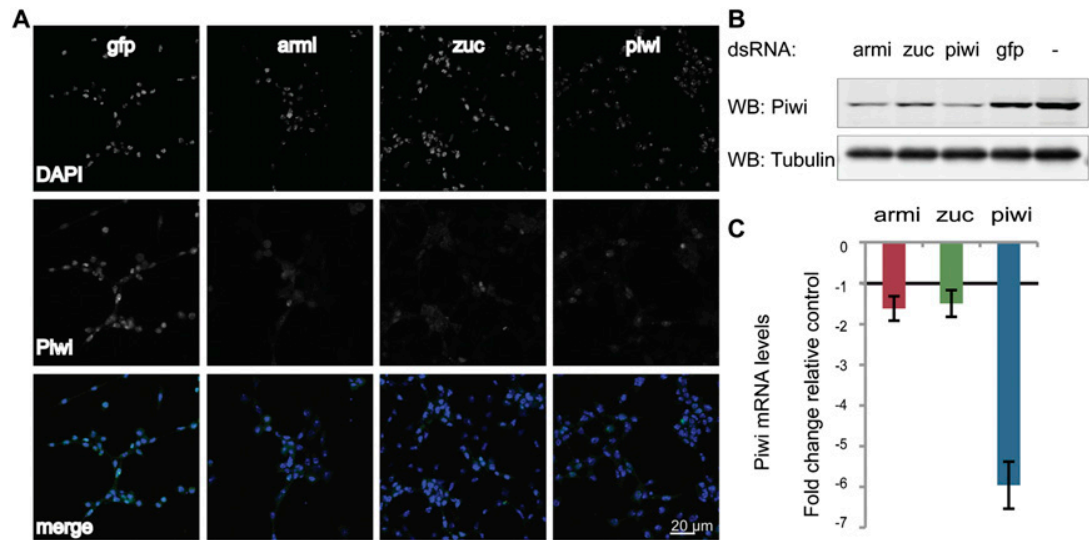

Figure 2. Piwi protein localization and levels upon knockdown of armitage or zucchini. (A) Piwi subcellular localization was determined by immunostaining in OSS cells treated with zuc-dsRNA, armi-dsRNA, or piwi-dsRNA. Gfp-dsRNA-treated cells were used as control. (B) Piwi protein levels in total cell extracts were determined by Western blotting. Tubulin was used as a loading control. (D) Piwi qPCRs were normalized to rp49. Fold changes relative to cell treated with gfp-dsRNA are shown on a linear scale. Error bars represent one standard deviation over three biological replicates.

a role in primary piRNA biogenesis, aiding piRNA production or loading, with this model resting on the presumption that association with mature piRNAs influences Piwi protein stability (Fig. 3A, steps 1, 2). Alternatively, Armi or Zuc could be core components of mature Piwi RISC, with loss of either subunit destabilizing associated components of the complex (Fig 3A, step 3).

\section{Armitage is a component of Piwi RISC}

To investigate these alternative models, we performed proteomic analysis of Piwi RNPs. Piwi immunoprecipitates contained a number of peptides from Armi, suggesting that this protein is present in Piwi RISC (Fig. 3B). Of note, we also detected association of both Piwi and Armi with Squash (Squ), another previously identified piRNA pathway component (Pane et al. 2007). Piwi could be also detected in Squ immunoprecipitates by Western blotting. Although no Zuc peptides were seen in multidimensional protein identification technology (MudPIT), Piwi could be detected to a low extent in Zuc immunoprecipitates (Fig. 3C; Supplemental Fig. S2). Overall, the emerging picture suggests that both Armi and Squ are components of Piwi RISC. Lower levels of Piwi associated with Zuc might indicate a weaker or more transient association of Zuc with Piwi RISC.

\section{Squash impacts the piRNA effector step}

Mutations in squash (squ) show little impact on piRNA populations in mutant ovaries (Malone et al. 2009). Similarly, upon sequencing of small RNAs in Piwi immunoprecipitates, we failed to detect any differences in associated piRNA populations upon comparison of squ homozygous mutant animals to heterozygous siblings (Fig. 4B). Animals harboring two squ alleles interrupted by early stop codons did, however, display an effect on transposon silencing.

As compared with heterozygous siblings, squ mutants showed significant derepression of gypsy (Fig. 5A). This occurred without any detectable change in an abundant gypsy piRNA or overall Piwi levels (Fig. 5B,C). In contrast, no substantial changes were detected in idefix or
ZAM (Fig. 5A,B); however, I-element and blood were strongly derepressed (Supplemental Fig. S3).

Considered together, these results point to a role of squash in the effector phase of the piRNA pathway. We did note a slight but reproducible reduction in Piwi protein levels in homozygous squ mutants (Fig. 5C; Supplemental Fig. S4). However, this was well within the range observed in Piwi heterozygotes, where the piRNA pathway functions completely normally.

\section{A possible role for Zucchini in piRNA biogenesis}

In the initial screen that placed zuc within the piRNA pathway, two alleles were identified (Schupbach and Wieschaus 1991; Pane et al. 2007). zuc ${ }^{\mathrm{HM} 27}$ represents an early stop mutation resulting in a putative null allele (referred to as zuc mut). This mutant strongly affects piRNA silencing in both germline and somatic cells of the ovary (Pane et al. 2007; Malone et al. 2009). While somatic piRNAs are depleted in this mutant, ping-pong signatures remain intact (Malone et al. 2009). This places Zuc outside of the adaptive phase, consistent with our accumulating evidence for a role in the initiation phase.

While the biochemical properties of Zuc have yet to be analyzed, its protein sequence places it as a member of the phospholipase D (PLD) family of phosphodiesterases. These share a $\operatorname{HxK}(x)_{4} \mathrm{D}$ motif, whose integrity is
A

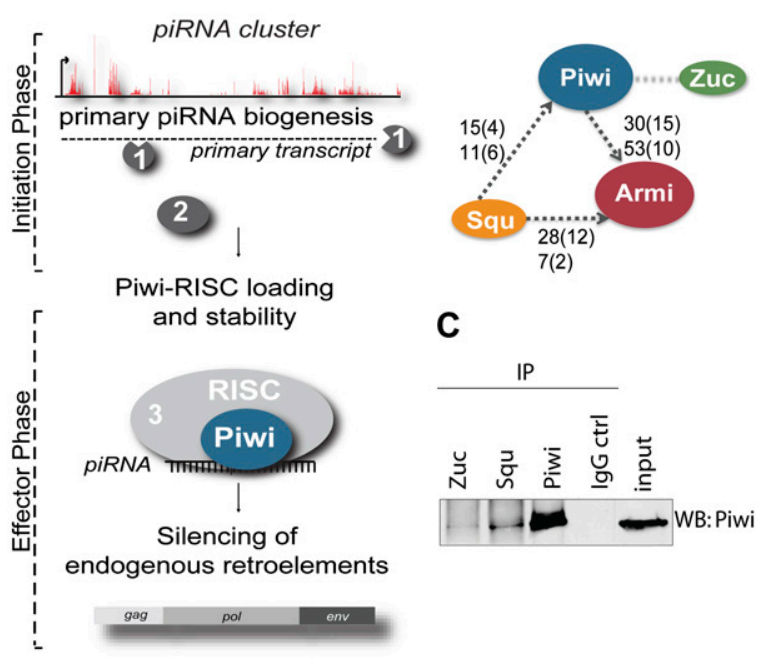

Figure 3. MudPIT analysis of Piwi, Zucchini, and Squash complexes. (A) A proposed model for the function of piRNA pathway components is shown. (B) Protein associations identified by MudPIT are depicted. Arrows point from the immunoprecipitated protein to the coimmunoprecipitated protein. Numbers of identified peptides and corresponding unique sequences (shown in parentheses) of two biological replicates are indicated. Only peptides above the significance threshold were considered (see the Materials and Methods). (C) Zucchini, Squash, and Piwi were immunoprecipitated (IP) from OSS cells. The presence of Piwi was assessed by Western blotting. 
Haase et al.
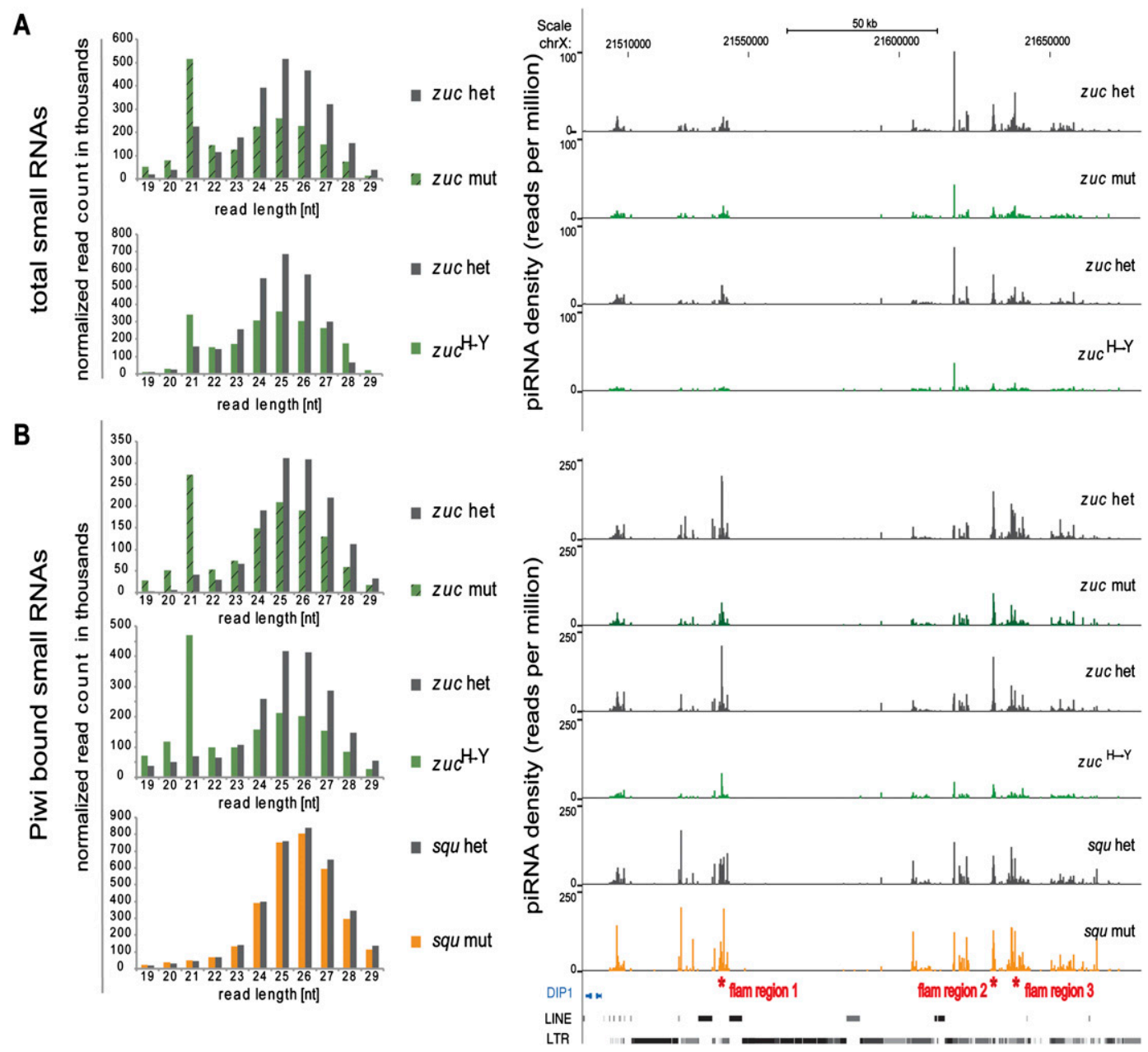

Figure 4. piRNA populations from zucchini and squash mutant ovaries. Heterozygous siblings serve as control. (Left panel) Size profiles of small RNAs mapping to repeats normalized to total read counts are shown. (Right panel) Densities of uniquely mapping piRNAs are plotted over the flamenco locus in reads per million (only small RNAs matching the plus strand are depicted). (A) Small RNAs cloned from total RNA. (B) Small RNAs from Piwi immunoprecipitates (IP). Regions of flamenco measured in Figure 5D are indicated by asterisks.

essential for catalytic activity (Zhao et al. 1997; Sasnauskas et al. 2010). The second zuc mutation that emerged in the original screen, $z u c^{\mathrm{SG} 63}$, contains a $\mathrm{H} \rightarrow \mathrm{Y}$ mutation within the phosphodiesterase motif that is predicted to render it catalytically inactive. To probe a role for Zuc catalytic activity in the piRNA pathway, we compared the presumed null (zuc mut) and catalytically dead (zuc $\mathrm{H} \rightarrow \mathrm{Y}$ ) alleles for their effects on piRNAs and transposon silencing.

We analyzed total ovarian small RNAs from animals that were heterozygous or homozygous for the zuc $\mathrm{H} \rightarrow \mathrm{Y}$ allele and compared the resulting profiles to previously published analyses of the presumed zuc mut allele (Malone et al. 2009). In both cases, we saw strong reductions in total piRNAs and in populations that mapped uniquely to the flamenco locus (Fig. 4A), regardless of the normalization method used to compare libraries (Supplemental Fig. S5). Slightly stronger impacts were apparent when we compared profiles of Piwi immunoprecipitates (Fig. 4B). Here, piRNA populations corresponding to flamenco were almost completely lost. We did note an accumulation of 21-nt species in Piwi immunoprecipitates from both zuc mutant lines. These were enriched for a $5^{\prime} \mathrm{U}$, although not to the extent for longer piRNA species. The nature of these shorter, apparently Piwiassociated RNAs remains mysterious.

Both the presumed null and $\mathrm{H} \rightarrow \mathrm{Y}$ zuc alleles impacted transposon silencing (Fig. 5A). Between fivefold and 20-fold increases in gypsy, ZAM, and idefix were noted in comparison with heterozygous controls. Even stronger derepression could be observed for I-element, HeT-A, 1731, and blood (Supplemental Fig. S3). The zuc $\mathrm{H}$ $\rightarrow$ Y and zuc mut alleles also showed similar impacts on piRNA populations (Fig. 5B) and the overall levels of Piwi protein (Fig. 5C; Supplemental Fig. S4).

Considered together, these data point to a requirement for the presumed catalytic center of Zuc in the initiation phase of the piRNA pathway. Other PLD family nucleases that have been characterized to date cleave nucleic acids leaving $5^{\prime}$ phosphate and $3^{\prime}$ hydroxyl termini (Pohlman et al. 1993; Zhao et al. 1997; Sasnauskas et al. 2010). These are the characteristics one might expect for a processing enzyme that catalyzed primary piRNA biogenesis. Previous studies have posited the requirement for several nucleolytic activities in the piRNA pathway. One is thought to form the $5^{\prime}$ ends of primary piRNAs. The 
A

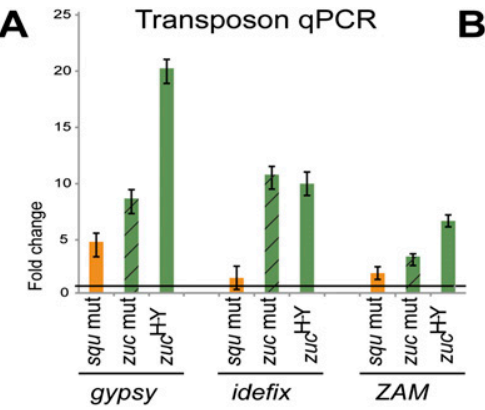

C

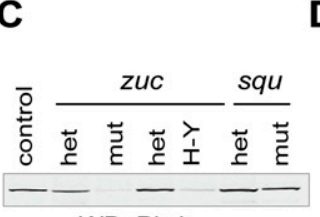

WB: Piwi

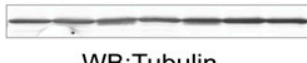

WB:Tubulin
B

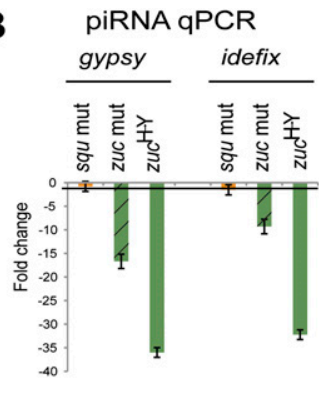

flamenco qPCR:

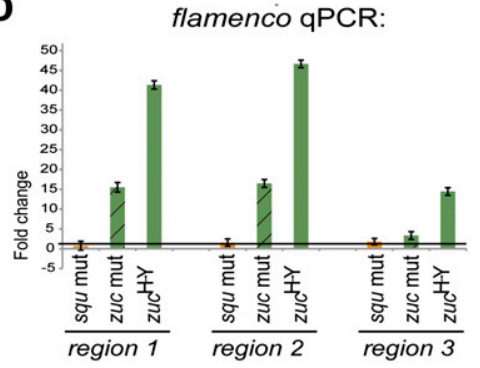

Figure 5. Transposons, piRNAs, and piRNA precursors in zucchini and squash mutant ovaries. (A) Transcripts of gypsy, idefix, and $Z A M$ transposons were detected by qPCR. (B) Individual piRNAs targeting gypsy and idefix were detected by qPCR. $(C)$ Piwi protein levels in mutant and heterozygous ovary extracts were measured by Western blotting. Tubulin serves as loading control. Celera sequencing strain (S-strain) is shown in addition. $(D)$ Three $\sim 100$-nt regions of flamenco that are normally highly processed into piRNAs were detected by qPCR. The positions of these segments are indicated in Figure 4. qPCR data were normalized to internal controls $r p 49(A, C)$ or bantam $(B)$. Fold changes relative to heterozygous siblings are shown on a linear scale. Error bars represent one standard deviation over three technical replicates.

$3^{\prime}$ ends of these species could be formed prior to Piwi loading or could be coupled to protein binding, as is posited for the ping-pong cycle. The nucleolytic center of Piwi proteins themselves form the $5^{\prime}$ ends of secondary piRNAs, with their $3^{\prime}$ ends proposed to be created by a separate enzyme. Based on its impacts in the soma on Piwi complexes, we imagined that the Zuc catalytic center might form either the $5^{\prime}$ or $3^{\prime}$ ends of primary piRNAs.

To evaluate this hypothesis, we examined RNAs derived from the flamenco locus in control ovaries or in tissues from animals homozygous for either of the two zuc mutant alleles. The prevailing model holds that the flamenco locus is transcribed as a continuous, singlestranded precursor spanning $>150 \mathrm{~kb}$ (Brennecke et al. 2007). We reasoned that a defect in primary processing might result in an accumulation of long RNAs from this locus, since they would not be effectively metabolized into piRNAs. By quantitative PCR (qPCR) using primer pairs spanning three different regions of flamenco (see Fig. 4B, right panel), we saw 15 -fold to 45 -fold increases in flamenco-derived long RNAs in zuc mutant ovaries (Fig. 5D; Supplemental Fig. S6).

Considered as a whole, our results strongly support a role for Zucchini in the primary processing of piRNAs from the flamenco locus. Given its size, it is virtually impossible to follow the fate of the intact flamenco transcript by Northern blotting. Three different segments of the locus do show an accumulation consistent with

their failure to be parsed into piRNAs. However, several alternative explanations can also be envisioned. For example, if Zucchini impacts Piwi stability, feedback controls might operate to inhibit primary biogenesis. Without a direct, biochemical demonstration that Zucchini processes piRNA cluster transcripts, its assignment as a primary biogenesis enzyme must be viewed as provisional. However, any alternative model must account for the requirement for its phosphodiesterase active site, and, at present, a direct role in piRNA biogenesis seems the most parsimonious conclusion.

While our studies do not ascribe specific functions to Armitage and Squash, they do support their assignment to the initiator and effector phases, respectively. Armitage is a putative helicase, although no analyses as yet indicate whether this biochemical activity is required for its function. Our placement of this protein in the initiation phase and its intimate association with Piwi perhaps suggest a role in loading or stability of Piwi RISC. Squash, of all the components examined in this study, had the most variable effects on transposon control in somatic cells of the ovary (Figs. 1A, 5A), but both its physical association with Piwi RISC and its impact on transposons without an effect on piRNAs imply a role in the effector phase. While the studies reported herein can suggest roles for known pathway components at specific points in the piRNA pathway, a definitive conclusion regarding the part played by any of these proteins will require reconstitution of the pathway in vitro.

\section{Materials and methods}

\section{OSS cell culture and knockdown}

The OSS cell line was a kind gift from Yuzo Niki, and was cultured as described (Niki et al. 2006). dsRNA was prepared as described (http:// www.flyrnai.org/DRSC-PRS.html). Cells were transfected with dsRNA using Xfect Transfection Reagent (Clontech). Six days after transfection, RNA and protein were extracted. Detailed information for RT-qPCR, Western blotting, and immunofluorescence is available in the Supplemental Material.

\section{Fly stocks and allelic combinations}

Fly stocks and allelic combinations used were squ mut: squHE47/PP32 (Pane et al. 2007); zuc mut: zucHM27/Df(2I)PRL (Pane et al. 2007); and zuc $H \rightarrow$ Y: zucHM27/SG63 (Pane et al. 2007).

\section{Preparation of small RNA libraries}

Small RNA libraries were prepared as described (Brennecke et al. 2007). Detailed information is available in the Supplemental Material.

\section{Acknowledgments}

We thank Vasily Vagin, Anna Jankowska, Adam Rosebrock, Yaniv Erlich, Julius Brennecke, Alexei Aravin, Delphine Fagegaltier, and current and former members of the Hannon laboratory for helpful discussions and support. The OSS cell line was a gift of Yuzo Niki (Ibaraki University). Fly stocks were kindly provided by Gertrud Schupbach (Princeton). PMG is supported by Grant 5T32GM065094 from the NIH and by a William Randolph Hearst Foundation Scholarship. S.F. is supported by the Elizabeth Sloan Livingston Foundation. B.C. is supported by the Boehringer Ingelheim Fonds. G.J.H. is an investigator of the Howard Hughes Medical Institute. This work was supported by grants from the NIH (to G.J.H.). Sequences reported in this study can be found at GEO using accession number GSE24108 (which includes libraries GSM593297-593304). 


\section{References}

Aravin A, Gaidatzis D, Pfeffer S, Lagos-Quintana M, Landgraf P, Iovino N, Morris P, Brownstein MJ, Kuramochi-Miyagawa S, Nakano T, et al. 2006. A novel class of small RNAs bind to MILI protein in mouse testes. Nature 442: 203-207.

Aravin AA, Hannon GJ, Brennecke J. 2007. The Piwi-piRNA pathway provides an adaptive defense in the transposon arms race. Science 318: $761-764$.

Brennecke J, Aravin AA, Stark A, Dus M, Kellis M, Sachidanandam R, Hannon GJ. 2007. Discrete small RNA-generating loci as master regulators of transposon activity in Drosophila. Cell 128: 10891103.

Brennecke J, Malone CD, Aravin AA, Sachidanandam R, Stark A, Hannon GJ. 2008. An epigenetic role for maternally inherited piRNAs in transposon silencing. Science 322: 1387-1392.

Brower-Toland B, Findley SD, Jiang L, Liu L, Yin H, Dus M, Zhou P, Elgin SC, Lin H. 2007. Drosophila PIWI associates with chromatin and interacts directly with HP1a. Genes Dev 21: 2300-2311.

Cook HA, Koppetsch BS, Wu J, Theurkauf WE. 2004. The Drosophila SDE3 homolog armitage is required for oskar mRNA silencing and embryonic axis specification. Cell 116: 817-829.

Cox DN, Chao A, Lin H. 2000. piwi encodes a nucleoplasmic factor whose activity modulates the number and division rate of germline stem cells. Development 127: 503-514.

Ghildiyal M, Zamore PD. 2009. Small silencing RNAs: An expanding universe. Nat Rev Genet 10: 94-108.

Girard A, Sachidanandam R, Hannon GJ, Carmell MA. 2006. A germlinespecific class of small RNAs binds mammalian Piwi proteins. Nature 442: 199-202.

Grivna ST, Beyret E, Wang Z, Lin H. 2006. A novel class of small RNAs in mouse spermatogenic cells. Genes Dev 20: 1709-1714.

Gunawardane LS, Saito K, Nishida KM, Miyoshi K, Kawamura Y, Nagami T, Siomi H, Siomi MC. 2007. A slicer-mediated mechanism for repeat-associated siRNA $5^{\prime}$ end formation in Drosophila. Science 315: $1587-1590$.

Klattenhoff C, Xi H, Li C, Lee S, Xu J, Khurana JS, Zhang F, Schultz N, Koppetsch BS, Nowosielska A, et al. 2009. The Drosophila HP1 homolog Rhino is required for transposon silencing and piRNA production by dual-strand clusters. Cell 138: 1137-1149.

Klenov MS, Lavrov SA, Stolyarenko AD, Ryazansky SS, Aravin AA, Tuschl T, Gvozdev VA. 2007. Repeat-associated siRNAs cause chromatin silencing of retrotransposons in the Drosophila melanogaster germline. Nucleic Acids Res 35: 5430-5438.

Lau NC, Seto AG, Kim J, Kuramochi-Miyagawa S, Nakano T, Bartel DP, Kingston RE. 2006. Characterization of the piRNA complex from rat testes. Science 313: 363-367.

Lau NC, Robine N, Martin R, Chung WJ, Niki Y, Berezikov E, Lai EC. 2009. Abundant primary piRNAs, endo-siRNAs, and microRNAs in a Drosophila ovary cell line. Genome Res 19: 1776-1785.

Li C, Vagin VV, Lee S, Xu J, Ma S, Xi H, Seitz H, Horwich MD, Syrzycka M, Honda BM, et al. 2009. Collapse of germline piRNAs in the absence of Argonaute3 reveals somatic piRNAs in flies. Cell 137: 509-521.

Malone CD, Hannon GJ. 2009. Small RNAs as guardians of the genome. Cell 136: 656-668.

Malone CD, Brennecke J, Dus M, Stark A, McCombie WR, Sachidanandam R, Hannon GJ. 2009. Specialized piRNA pathways act in germline and somatic tissues of the Drosophila ovary. Cell 137: 522-535.

Niki Y, Yamaguchi T, Mahowald AP. 2006. Establishment of stable cell lines of Drosophila germ-line stem cells. Proc Natl Acad Sci 103: 16325-16330.

Olivieri D, Sykora M, Sachidanandam R, Mechtler K, Brennecke J. 2010. An in vivo RNAi assay identifies major genetic and cellular requirements for primary piRNA biogenesis in Drosophila. EMBO I doi: 10.1038/emboj.2010.212.

Pal-Bhadra M, Leibovitch BA, Gandhi SG, Rao M, Bhadra U, Birchler JA, Elgin SC. 2004. Heterochromatic silencing and HP1 localization in Drosophila are dependent on the RNAi machinery. Science 303: 669672.

Pane A, Wehr K, Schupbach T. 2007. zucchini and squash encode two putative nucleases required for rasiRNA production in the Drosophila germline. Dev Cell 12: 851-862.
Pohlman RF, Liu F, Wang L, More MI, Winans SC. 1993. Genetic and biochemical analysis of an endonuclease encoded by the IncN plasmid pKM101. Nucleic Acids Res 21: 4867-4872.

Saito K, Nishida KM, Mori T, Kawamura Y, Miyoshi K, Nagami T, Siomi H, Siomi MC. 2006. Specific association of Piwi with rasiRNAs derived from retrotransposon and heterochromatic regions in the Drosophila genome. Genes Dev 20: 2214-2222.

Saito K, Inagaki S, Mituyama T, Kawamura Y, Ono Y, Sakota E, Kotani H, Asai K, Siomi H, Siomi MC. 2009. A regulatory circuit for piwi by the large Maf gene traffic jam in Drosophila. Nature 461: 1296-1299.

Sarot E, Payen-Groschene G, Bucheton A, Pelisson A. 2004. Evidence for a piwi-dependent RNA silencing of the gypsy endogenous retrovirus by the Drosophila melanogaster flamenco gene. Genetics 166: 13131321.

Sasnauskas G, Zakrys L, Zaremba M, Cosstick R, Gaynor JW, Halford SE, Siksnys V. 2010. A novel mechanism for the scission of doublestranded DNA: BfiI cuts both $3^{\prime}-5^{\prime}$ and $5^{\prime}-3^{\prime}$ strands by rotating a single active site. Nucleic Acids Res 38: 2399-2410.

Schupbach T, Wieschaus E. 1991. Female sterile mutations on the second chromosome of Drosophila melanogaster. II. Mutations blocking oogenesis or altering egg morphology. Genetics 129: 1119-1136.

Vagin VV, Sigova A, Li C, Seitz H, Gvozdev V, Zamore PD. 2006. A distinct small RNA pathway silences selfish genetic elements in the germline. Science 313: 320-324.

Zhao Y, Stuckey JA, Lohse DL, Dixon JE. 1997. Expression, characterization, and crystallization of a member of the novel phospholipase D family of phosphodiesterases. Protein Sci 6: 2655-2658. 


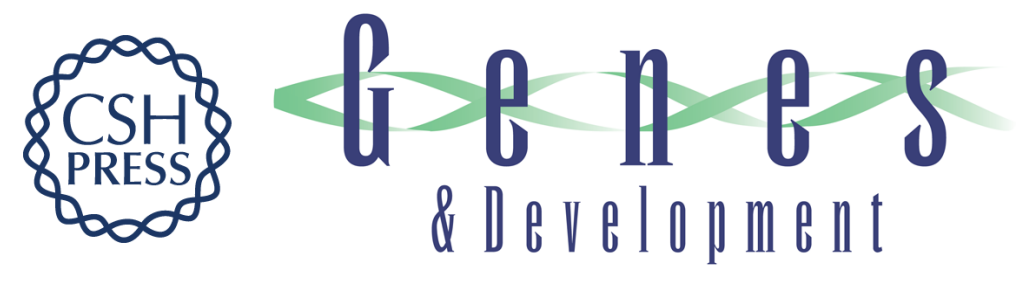

\section{Probing the initiation and effector phases of the somatic piRNA pathway in Drosophila}

Astrid D. Haase, Silvia Fenoglio, Felix Muerdter, et al.

Genes Dev. 2010, 24: originally published online October 21, 2010

Access the most recent version at doi:10.1101/gad.1968110

\section{Supplemental http://genesdev.cshlp.org/content/suppl/2010/10/15/gad.1968110.DC1 Material}

References This article cites 29 articles, 12 of which can be accessed free at: http://genesdev.cshlp.org/content/24/22/2499.full.html\#ref-list-1

\section{License}

Email Alerting

Receive free email alerts when new articles cite this article - sign up in the box at the top Service

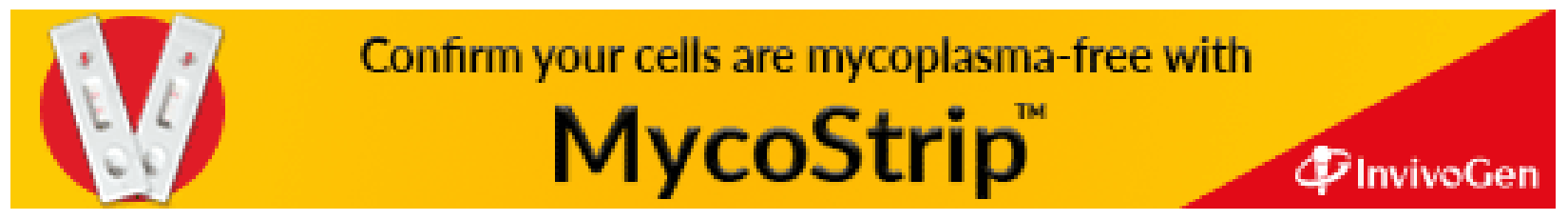

